\title{
ICN Based Shared Caching in Future Converged Fixed and Mobile Network
}

\author{
Zhe Li, \\ Jean-Charles Point \\ JCP-Connect, Rennes, France \\ zhe.li@jcp-connect.com \\ pointjc@jcp-connect.com
}

\author{
Selami Ciftci, Onur Eker \\ Argela, Ankara, Turkey \\ Firstname.Lastname@argela.com.tr
}

\author{
Giulia Mauri, Marco Savi, \\ Giacomo Verticale \\ Politecnico di Milano, Italy \\ firstname.lastname@polimi.it
}

\begin{abstract}
The explosion of mobile multimedia and Internetof-things (IoT) services implies strong requirements for seamless switching among various types of networks. Thus, to offer true ubiquitous Internet connection, a Fixed and Mobile Converged (FMC) network architecture is essential for the future 5G network. Such a convergent network can not only improve the utilization of network resources, but also inspire new add-on services for FMC network operators. In this paper, we introduce a shared caching overlay based on Information Centric Networking (ICN). It is deployed on top of the FMC network and controlled by the FMC network operator to offer Caching as a Service (CaaS) to Over-The-Top (OTT) service providers and virtual network operators. Business analysis and performance evaluation will highlight the benefits of deploying such a controlled Shared Caching System (SCS) over an FMC network.
\end{abstract}

\section{INTRODUCTION}

The goal of future $5 \mathrm{G}$ network is to provide ubiquitous Internet connection and high quality network services. However, the current access and aggregation network architectures, where different network types are managed separately, prevent the emergence of various necessary features of $5 \mathrm{G}$ network. For example, seamless user experience when switching from one network type to another is hindered by distinct management models of subscriber data and session used in fixed broadband, mobile and Wi-Fi networks. Network infrastructure openness is still limited because of the isolation of different network infrastructures. This limits the programmability of the network and blocks the exploitation of network functionalities for OTT service providers and developer's communities. Consequently, nowadays telecommunication operators that own different network infrastructures are searching for a converged network architecture that is controlled through a unified management system. The novel network architecture will on the one hand maximize the utilization of network resources, and on the other hand enable new business models of resource bundled services for the FMC network operators to increase their revenue.

Various technical enablers, such as very tight coupling of LTE and Wi-Fi networks [1] and unified authentication, authorization, accounting, have been proposed to achieve such a convergent access and aggregation network. This paper investigates the feasibility of another indispensable enabler of future 5G network, namely the in-network caching technology in the FMC network, so as to create new business opportunities for FMC network operators and further improve the QoE/QoS at user side.

Our approach is to exploit the clean-slate Information Centric Networking (ICN) architecture, particularly the Content Centric Networking (CCN) [2], to construct a controlled shared caching overlay on top of the FMC access or aggregation network. ICN aims to shift the current complex Internet model to a simple and generic one. The basic networking unit is no longer the identified node (e.g., servers, routers, terminals) but the named content object. The ICN networking activities follow a receiver-driven model, where end-users only express their interests for a given content. Then, the entire network is in charge of routing the requests based only on content names towards the best content source and delivering the contents through the reverse paths to the end-users. The ICN paradigm natively includes features such as location-independent naming, name-based routing, native multicast, self-secured content, and most importantly, in-networking caching.

These built-in functionalities suit well the FMC network where unified routing protocol and management schemes should be applied to serve the user requests and guarantee the service quality and security. Indeed, the widespread use of in-network caching blends well with specific issues of the FMC network, such as seamless handover among different network infrastructures, and intermittent connectivity in a mobile environment. In this paper, an upgraded $\mathrm{CCN}$ protocol will be presented, which allows FMC network operators to easily construct and manage a Shared Caching System (SCS) and offers CaaS to OTT service providers or virtual network operators.

The rest of the paper is structured as follows: Section II briefly introduces the related work. We provide some background notions on CCN communication model and FMC in Section III We describe the architecture of CCN-based SCS for FMC network operators in Section IV. Section V shows preliminary numerical results obtained from a specific use case to highlight the benefit of SCS. Finally, Section VI concludes the paper and identifies business opportunities for such a shared caching system in FMC networks.

\section{RELATED WORK}

Since the end of the last century, substantial efforts have been put on content caching in various contexts of network services to reduce content access delay. One of the greatest 
success is the commercialization and wild deployment of Content Delivery Network (CDN) in Internet [3, 4]. Nevertheless, since CDN surrogates are located at the edge of the IP network, they are not able to alleviate the burden in the access segment of fixed and mobile networks. As a consequence, a more deeply integrated in-network caching system is gaining a lot of attention from both industry and academia. For example, innetwork caching is enabled on various wireless network equipments such as Acess Point (AP) in Wi-Fi networks [5], eNodeB (eNB) in LTE networks [6] as well as femtocell box [7] to offload video traffic from mobile backhaul. Caching solutions for $3 \mathrm{G}$ or LTE networks have been commercialized by companies like Allot Communications, PeerApp, Altobridge, etc. In [8], the authors proposed a hierarchical in-network caching architecture with storage deployed in the Evolved Packet Core, in the Radio Access Network (RAN) and even in end devices that form a Device-to-Device collaborative caching network. Recent studies [9] have preliminarily discussed the expected gains of a cooperative caching in both LTE and WiFi networks in the case that a dual attachment for User Equipments (UEs) is enabled.

In the context of ICN, in-network caching management is also an attractive topic. Various studies have proposed advanced caching protocols [10]-[12]. The authors in [10] propose an optimal content placement policy for an IPTV system in a hierarchical tree-like topology. For the same IPTV service, the authors of [11] realize the cooperative caching by jointly considering the content placement and dynamic request routing. In [12], the authors design a cooperative in-network caching protocol for catch-up TV service in CCN. The authors of [13] focus on a mobility management scheme in CCN. It evaluates the routing update latency and the delivery latency depending on the number of nodes. The results show that the mobile CCN scheme achieves better performance than the basic $\mathrm{CCN}$ protocol. A proactive caching algorithm for Named Data Network (NDN) is presented in [14]. The algorithm pre-allocates the content according to user requirements and mobility information by modifying the request message and the basic communication protocol. More recently, an optimal content placement scheme for vehicular network is reported in [15]. The work formulates the optimal content distribution problem using an ILP model and shows how a vehicular network can exploit an ICN architecture to maximize the probability to retrieve the desired content at user side.

In our work, we propose a unified management scheme of CCN caching for FMC network, which takes into account not only the user mobility but also other requirements such as traffic offloading, application-aware content placement, etc. The storage resource in this controlled caching system can be shared by OTT service providers and regarded as a service offered by FMC network operator.

\section{BACKGROUND}

This section first recalls how the $\mathrm{CCN}$ architecture works, then it shows how network and content management are performed in a FMC network.

\section{A. CCN Communication Model}

In $\mathrm{CCN}$, the information exchange is realized by two types of packets: Interest and Data. An Interest packet represents the content request from an end user, while the Data packet carries the corresponding content. Both the Interest and the Data packets are identified by an URI alike Content Name that is used for routing and caching. A Data packet is sent back as a response to an Interest packet only if their Content Names match.

The in-network caching in $\mathrm{CCN}$ is performed by the Content Store (CS). Once an Interest arrives at a CCN node, a longest-prefix match lookup on Content Name is executed to firstly check if the required content exists already in the CS. If the corresponding content is found in the CS, the Data packet is sent out directly through the interface that has received the request and the Interest is dismissed. Otherwise, the $\mathrm{CCN}$ node looks for the Content Name in a table called Pending Interest Table (PIT), which keeps track of the unsatisfied Interest packets and their arrival interface. It ensures that the content can be sent back to the end user via the reverse path of the forwarded Interest. If the Content Name is found in the PIT, the arrival interface is added to the entry, and the Interest is discarded. While if there is not a match in the PIT, the Interest is tackled by the Forwarding Information Base (FIB), which is a Content Name based routing table containing all the potential content sources. It is worth mentioning that the FIB is filled when a new content appears in the network. Content providers register the prefix of the content they can provide to the CCN node they connect to. The registered prefix is then put into link state advertisement (LSA) and propagated to other CCN nodes. If a FIB match is available, the Interest is sent out to all the interfaces registered in the corresponding FIB entry, and a new entry is inserted in the PIT. Finally, if a match is not found, the Interest is discarded because the node does not know how to find any matching Data. The processing of a Data packet is relatively simple. Only when there is a PIT match, the data packet is sent out over all the interfaces registered in the entry and the content is stored in the CS. Otherwise, the packet is discarded, since it is either duplicated or unsolicited.

While in-network caching is intrinsically implemented in $\mathrm{CCN}$, only transparent caching is offered in the original setting of the CCNx daemon ${ }^{1}$. The control on content cache in $\mathrm{CCNx}$ is mainly realized by specifying in the per-hop header [16] two options: i) do not cache the content, and ii) MaxAge that indicates the validity of a content. The first is a constraint on cache, which can use the content only to satisfy the pending interest but cannot store it. While, the latter is a constraint on data and defines its expiration. After MaxAge has lasted, the content is automatically evicted from the caches. In our controlled caching system, we will introduce more sophisticated management schemes.

\section{B. Network and content management in an FMC network}

In this work, we assume that a FMC network is deployed. Such network, characterized by fixed/mobile unified access and aggregation segments, is shown in Fig. 1. The most important functional node in this FMC architecture is the Unified Access Gateway (UAG). The UAG is a network element in charge of logically supporting the service creation for both fixed and mobile users. Its control plane is composed of two different

\footnotetext{
${ }^{1}$ Software implementation of $\mathrm{CCN}$ over IP network
} 


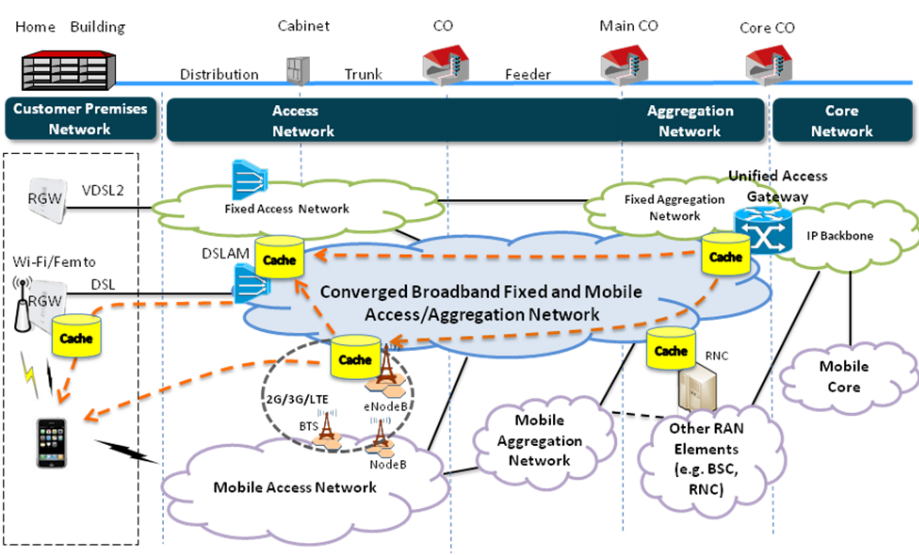

Fig. 1: FMC Framework

management layers: the network-management layer and the content-management layer. The network-management layer deals with traffic-related functions (e.g., topology management and routing), while the content-management layer handles all the content-related functions (e.g., cache and metadata management).

The introduction of a content-management layer in the UAG increases the flexibility for mobile and fixed operators in their business relationship with content providers. In fact, the network-management layer of the UAG could be simply controlled by network operators while its content-management layer could be controlled either by the network operator or by the content provider, as shown in Fig. 2. Regarding the content management, one option would be to let the network operator control the UAG and to have only one entity running the UAG control plane. Another option would be to let different operators control different management layers. In this way, each operator optimizes content- and networking-related functions by exploiting its own expertise.

In the specific, as we will show in the following section, the adoption of an ICN overlay scheme based on the $\mathrm{CCN}$ protocol, allows to dramatically simplify the content management in an FMC network.

\section{CCN-BASED ShaRed CACHING IN FMC}

The UAG acts as the universal coordinated convergence controller in the FMC networks. In this respect, the UAG is the main interaction entity in the network between FMC network operator and other stakeholders. Therefore, the UAG is also the heart of the caching framework in FMC networks, as it does not only store the cached content, but it is also responsible for the delivery to end users of content cached at any FMC node. For example, in a vertical 4G-to-Wi-Fi handover scenario, the content cached at UAG, Wi-Fi access points and eNodeBs, can be managed/delivered via intelligent pre-fetching or any other caching technique without the need for any content transmission in the core network. According to this example, the content-management layer takes decisions on where to cache the content using its cache and metadata management modules, while the network-management layer deals with transmission of the content from the caching location to eNodeB or Wi-

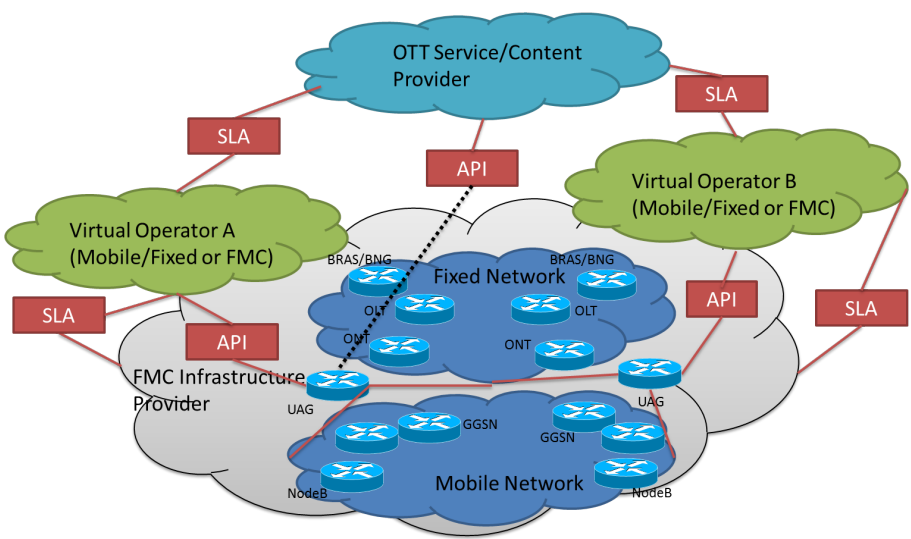

Fig. 2: Caching Based Network Sharing Scenarios for FMC Networks

Fi access point using its topology and routing management modules, transparently to the end users.

To realize content management, a cache controller is integrated in the UAG. The cache controller (CC) receives inputs from both content providers and network operators, and gives as outputs optimal caching and real-time pre-fetching plans. Directives will be sent to the caching nodes in the CCN caching overlay to execute these plans. The architecture of the CCN based SCS is shown in Fig. 3.

All the content traffic carried by HTTP protocol passes through the caching overlay. This can be done by intercepting the TCP packets sent via port 80 . Once a HTTP content request is received by a caching node, it is transformed into a $\mathrm{CCN}$ Interest. Then, the Interest is processed by the CCN protocol as it is described in Section III-A. If none of the caching nodes can satisfy the request, it is forwarded to the caching node connected to the UAG. Then, a conversion from CCN Interest to a normal HTTP request is performed and the request is forwarded to the IP network. Once a caching node is installed in the network, it sends heart-beat messages directly through the IP network at a certain frequency to the $\mathrm{CC}$ to indicate its presence in the network and its correct functioning. This way, the CC can keep the identifier (e.g., the IP address) of each caching node in order to send management directives.

\section{A. Updated CCN protocol}

A Local Management Primitive (LMP) is added to CCNx daemon (software) to execute the directives given by the $\mathrm{CC}$ to the CCN caching nodes. The LMP starts with the activation on a caching node. It registers its $\mathrm{CCN}$ prefix containing LMP_Prefix by sending a Register message to the CCNx daemon so that control directives can be forwarded to the LMP without any problem. This Register message will not be propagated to ensure that only the LMP and the CCNx daemon located on the same caching node can communicate with each other.

Besides the global control on the content cacheability and lifetime in the caching overlay, we suggest to use another type of message, namely the Config packet, to carry the directives 


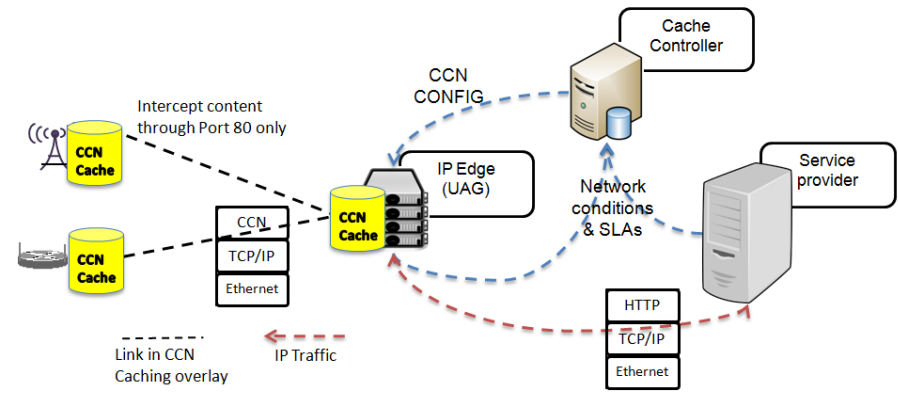

Fig. 3: CCN based SCS in FMC

for cache replacement policy and content pre-fetching management. When the $\mathrm{CC}$ receives inputs from the service provider and the FMC network operator, it sends a Config message with two sub-types indicated by its prefix, one for transparent caching management, the Config/Control packet, and the other for pre-fetching management, the Config/Prefetch. This message is directly sent to the destination caching node (through IP routing, with $\mathrm{CCN}$ port) although it is a $\mathrm{CCN}$ formatted message. When the $\mathrm{CCN}$ node receives this message, it forwards it to its unique LMP. Then, different actions can be taken according to the sub-type of the message.

1) Transparent Caching Management: The Config/Control message, for transparent caching management, carries an integer value indicating which caching policy should be used. The possible values are defined in Table I.

\begin{tabular}{|c|c|}
\hline Integer value & Caching policy \\
\hline \hline $0 \times 00$ & no caching allowed \\
\hline $0 x 01$ & random replacement \\
\hline $0 x 02$ & Least Recently Used (LRU) \\
\hline $0 \times 03$ & Least Frequently Used (LFU) \\
\hline
\end{tabular}

TABLE I: The Config/Control message

Additional fields, like usage_timestamp for LRU and usage frequency for LFU, are added to each content stored in the CS to remember the latest time when the content is required and the number of the requests for the content. The values of these fields are updated accordingly every time a request is received. To avoid a reduction in the speed of the routing process, the update action is done in an asynchronous manner. The integer value in the Config/Control message is saved in a variable called policy_indicator. Once policy_indicator is set to $0 x 00$ the CS size is set to 0 . Otherwise, when the CS is full, it eliminates the content according to the rules shown in Table I.

In the performance section, we assume that the caching policy remains the same for the duration of the simulation. However, the paper [17] evaluates and compares LRU, LFU and pre-fetching policies in a tree-based scenario, and it can be used as reference for our work.

2) Pre-fetching management: The Config/Prefetch message, in case of pre-fetching management, carries the name

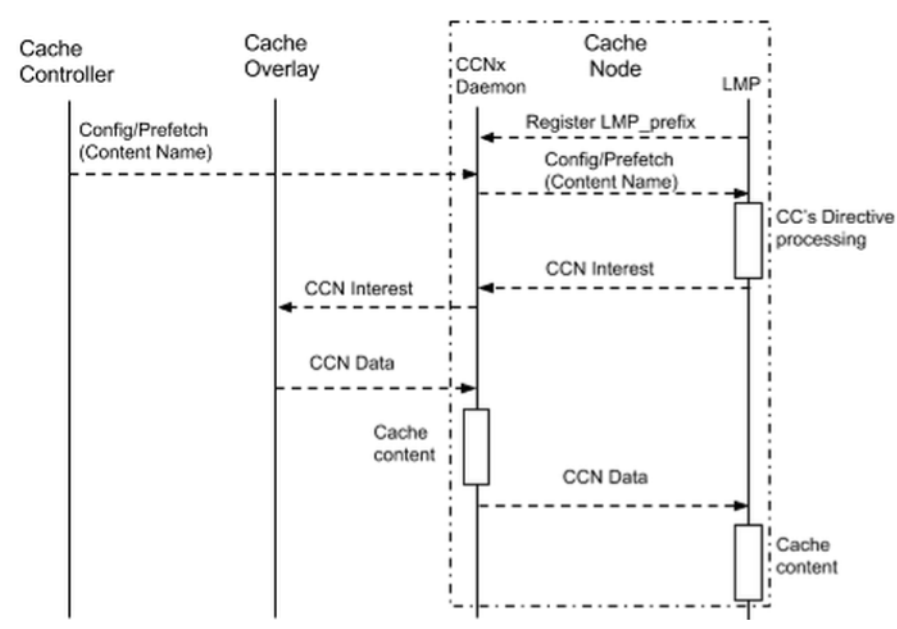

Fig. 4: Pre-fetching process

of the content that should be pre-fetched. Once the message is received by the LMP, it sends a standard CCN Interest to the CCNx daemon. The whole process is described in Fig. 4. The Cache Overlay on the figure represents any caching node in the network as well as the content source. Once the content has been found, it will be sent back in a CCN Data packet, and cached on every forwarding node. The Data packet is forwarded also to the LMP as corresponding response of the first Interest sent, and kept in a specific cache managed by LMP. The content will stay in the specific cache for a certain period of time so that the content will be available on the cache node even if it is evicted from CS. Note that in the diagram, only one Interest is presented, although in reality it is a stream of Interest packets sent by the caching nodes. When the user's Interest asking for the pre-fetched content arrives at the caching node, it may be directly satisfied by the CS in the CCNx daemon. If the content has already been evicted from the CS according to certain replacement policy, the Interest is sent on the interfaces towards all the potential content sources including LMP.

\section{Performance Evaluation}

This section evaluates the performance of such a shared caching system in terms of user side QoE/QoS as well as the system overhead. The whole network is abstracted to a CCN based caching overlay that concentrates on content delivery. We simulate the network using OMNET++. The caching node is built upon the CCN router used in [12] plus the LMP module.

\section{A. Test Scenario and Network Setup}

We assume that the FMC operator has a full control of the network, and it is able to obtain all the traces of user's motion information. That is, the operator knows when a user will change its Wi-Fi interface to LTE interface (and vice versa), and which access node it will attach to. Based on this information, content could be pre-fetched by the right caching node, but the pre-fetching will only be performed when the end user connects to a new access node. On the contrary, since 


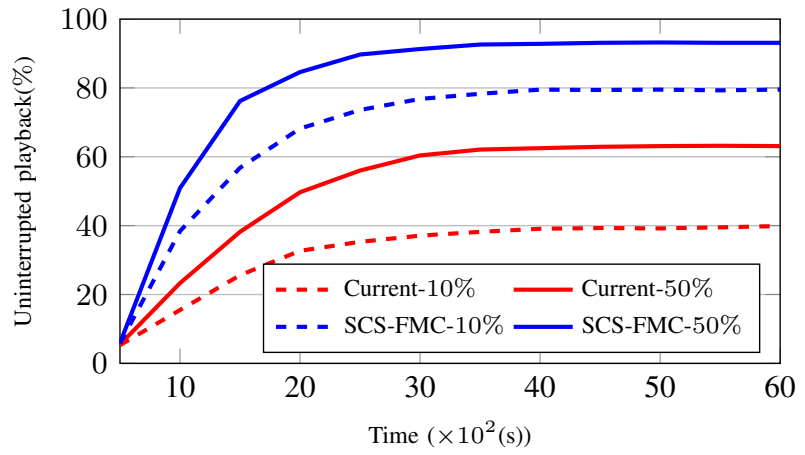

Fig. 5: Playback continuity

current mobile and Wi-Fi networks are managed separately, the mobile operator and the Wi-Fi operator do not have the same knowledge as the FMC operator so that they cannot detect the interface switching action of a UE. Therefore, only transparent caching can be performed in the cache overlay on top of current network. Concerning the interface selection, when the UE is covered by both Wi-Fi and LTE, it will select the Wi-Fi network as preference.

The test scenario is related to the example described in section IV. We used BonnMotion [18] to generate the random street map and the random walk model for 100 active mobile users. We assumed that the mobile users consume short movies with the sizes ranged from $10 \mathrm{MB}$ (a 5 minutes, $400 \mathrm{kbps}$ video) to $60 \mathrm{MB}$ (a 20 minutes video). Every video is divided into several clips of 5 seconds each. There are totally 10,000 videos in the content pool whose popularity follows a Zipf distribution with $\alpha=0.8$. When the UE moves, it will switch between LTE and Wi-Fi network according to the coverage of Wi-Fi APs. At the same time, the FMC network operator monitors the user behavior. Once it detects the possible switch from one eNB or AP to another, the SCS can pre-fetch the content before the switch happens so that when the user connects to the new access point, the content is already accessible in the local cache. For transparent caching in FMC SCS, we implement the LFU policy in the edge caching nodes and LRU policy in the centralized cache nodes following the results given in [19]. We assume that only the LRU policy is exploited in the caching system of the current network. On the other side, the pre-fetched content in LMP will be deleted right after the corresponding request is satisfied.

We deployed 8 APs and 2 eNBs over a geographical space around $2 \mathrm{~km}^{2}$. The APs' maximum link speed is $72 \mathrm{Mbps}$, and it serves simultaneously around 10 users on average. Therefore the link speed between APs and end users is fast enough so that if a video is cached in the AP, the user can obtain it immediately. Two eNBs are deployed in the network to provide a full coverage of the territory. However, they will be connected by UE only when a Wi-Fi network is not available. The average mobile link speed is set to $1 \mathrm{Mbps}$, which is also fast enough to deliver video chunk without any delay. We do not put any bandwidth limitation on backhaul links since it is not an issue in $\mathrm{CCN}$ [2]. However, we set the transmission latency to $0.5 \mathrm{~s}$ between two caching nodes. When a user request (not a prefetching request) has to be forwarded to IP network, the RTT

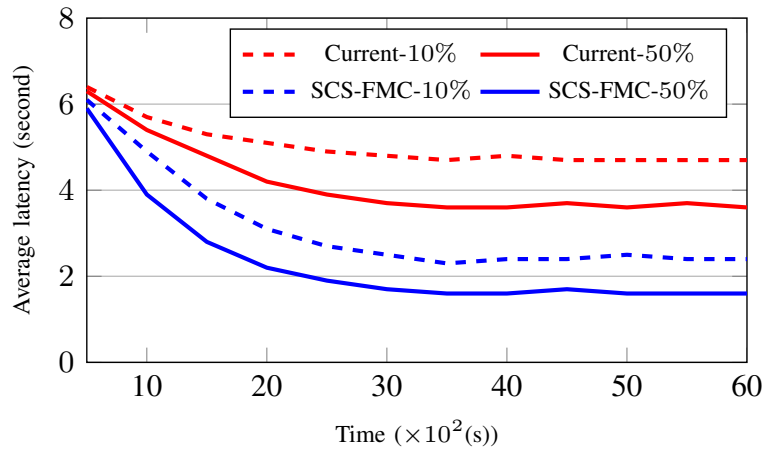

Fig. 6: Average Latency

of retrieving the video chunk from original content server is set to $5 s$. The local buffer on UE is set to one video chunk, that is $5 s$ playback. Thus, an RTT between UE and original content server will introduce an interruption of video playback at the user side. The total size of the equipped cache varies from $10 \%$ of the size of the total video data (around $80 \mathrm{~GB}$ ) to $50 \%$ of it (around $400 \mathrm{~GB}$ ). The duration of our simulation was one hour and a half, and there is no video stored in caching nodes at the beginning of the simulation.

\section{B. Simulation Results}

Fig. 5 displays the video playback continuity in different systems. Please note that in the current network setting, there is no pre-fetching function available. The results show an obvious advantage of FMC when the storage space in the cache overlay is relatively small. Compared to Current $-10 \%$, the percentage of playback continuity in SCS-FMC- $10 \%$ is doubled and reaches $80 \%$, which means $80 \%$ of the videos watched by the users in the network can be finished without any interruption. In our SCS setting, we do not consider any collaborative caching strategy, and deploy only a two levels hierarchical caching overlay. More gain on playback continuity can be envisaged in a collaborative caching system. The curves show also that increasing the cache size of SCS can further improve the QoS/QoE at user side. When the cache size is raised to $50 \%$ of the total content volume, SCS deployed in FMC can guarantee the continuity of $95 \%$ of the video sessions. It implies a very high cache hit-ratio in a controlled caching system.

The average latency to retrieve a video chunk is given in Fig. 6. The results fit well to the video playback continuity revealed in Fig. 5. The average latency in SCS-FMC-50\% is less than $2 s$, which means that most of the video chunks can be obtained from the caching in FMC network rather than from original content server.

We evaluate the system overhead by counting the packets processed by the UAG. The comparison between Current- $50 \%$ and SCS-FMC-50\% is given in Table II. Since the UAG does not exist in the current network setting, we take the number of packets received and sent by the original content server as a reference. The statistics show that UAG receives less Interest packets in FMC-SCS than the original server in current network. Since the number of user requests is almost the same in the tests for the two network settings, we can 


\begin{tabular}{|c|c|c|}
\hline $\begin{array}{c}\text { Network } \\
\text { configuration }\end{array}$ & $\begin{array}{c}\text { Interest packets received } \\
\text { by original content server } \\
\text { or UAG }\end{array}$ & $\begin{array}{c}\text { Packets sent } \\
\text { toward } \\
\text { access network }\end{array}$ \\
\hline \hline Current-50\% & 1 & 1 \\
\hline FMC-SCS-50\% & 0.88 & 1.23 \\
\hline
\end{tabular}

TABLE II: Number of Requests received and total packets sent by the $U A G$ (normalized by the number in Current $50 \%$ )

deduce that the gain on caching efficiency comes from the different replacement policies applied in the SCS but not in the current network. This highlights the benefits of deploying a controlled SCS against only a transparent caching system. On the other side, due to the control message, the UAG sends more packets toward the caching overlay than the original server. However, the volume of message stays in the same order, which indicates an acceptable overhead.

\section{CONCLUSION AND Business OpPoRTUNities}

As generally agreed, FMC will be the network architecture of the future. In this paper, we described an ICN based Shared Caching System, which allows FMC operator to offer CaaS to service providers and virtual network operators. The performance of the SCS is compared with a transparent caching system in the current network setting. Our results show that FMC network can significantly improve the caching efficiency as well as the QoS/QoE at the user side with reasonable overhead.

With convergent solutions, FMC networks have distinguished features that can be exploited for content-based services. Exploiting the varying storage capabilities of the fixed and mobile network nodes and cost-effective delivery mechanisms, FMC network operators have a great advantage whether being in an access-network provider or in an infrastructure provider role, as highlighted in Section III-B. The core of the new business models offered by caching-enabled FMC networks, whether being in access-network provider or in infrastructure provider role, is in providing new content-based bundle services to virtual operators and OTT service/content providers. The cooperation with content provider could also solve the problem of caching encrypted content. In this way, FMC operators are able to provide advanced caching services to these operators. On top of this, $\mathrm{QoS} / \mathrm{QoE}$ based differential pricing can also generate new means to revenue opportunities for FMC operators. Differential pricing can be introduced by defining quality based tariffs exploiting the advanced content distribution mechanisms. Moreover, caching-based content delivery reduces the number of bits carried on transit links and on the whole network. With careful resource management, FMC networks will maximize network-resource utilization in a holistic manner. As a result, new business models targeting new revenue channels as well as bit transmission reduction mechanisms targeting lower CAPEX/OPEX will yield higher network average revenue per user (ARPU).

\section{ACKNOWLEDGMENTS}

The work leading to these results has received funding from the European Union's Seventh Framework Programme (FP7/2007-2013) under grant agreement NO. 317762 "COMBO project".

\section{REFERENCES}

[1] X. Lagrange, "Very tight coupling between lte and wi-fi for advanced offloading procedures," in Wireless Communications and Networking Conference Workshops (WCNCW), 2014 IEEE, April 2014, pp. 82-86.

[2] V. Jacobson, D. Smetters, J. Thornton, M. Plass, N. Briggs, and R. Braynard, "Networking named content," in ACM CoNEXT'09. New York, USA, 2009.

[3] Carbon60, "Akamai edge caching, routing and security services," http://www.carbon60.com/global-services/akamai-edge-cachingrouting-security-services/.

[4] netflex, "Netflix open connect content delivery for isps," https://openconnect.itp.netflix.com/deliveryOptions/index.html.

[5] H. Gomaa, G. G. Messier, R. Davies, and C. Williamson, "Peer-assisted caching for scalable media streaming in wireless backhaul networks," in IEEE Global Telecommunications Conference (GLOBECOM 2010), 2010.

[6] H. Ahlehagh and S. Dey, "Video caching in radio access network: Impact on delay and capacity," in IEEE Wireless Communications and Networking Conference (WCNC), 2012.

[7] N. Golrezaei, K. Shanmugam, A. G. Dimakis, A. F. Molisch, and G. Caire, "Femtocaching: Wireless video content delivery through distributed caching helpers," in IEEE INFOCOM, 2012.

[8] X. Wang, M. Chen, T. Taleb, A. Ksentini, and V. C. M. Leung, "Cache in the air: exploiting content caching and delivery techniques for $5 \mathrm{~g}$ systems," IEEE Communications Magazine, vol. 52, 2014.

[9] J. Hachem, N. Karamchandani, and S. Diggavi, "Coded caching for heterogeneous wireless networks with multi-level access," in arXiv:1404.6560, 2014.

[10] S. Borst, V. Gupta, and A. Walid, "Distributed caching algorithm for content distribution networks," in IEEE INFOCOM, 2010.

[11] J. Dai, Z. Hu, B. Li, J. Liu, and B. Li, "Collaborative hierarchical caching with dynamic request routing for massive content distribution," in IEEE INFOCOM, 2012.

[12] Z. Li and G. Simon, "Time-shifted tv in content centric networks: The case for cooperative in-network caching," in IEEE ICC, 2011.

[13] J. Lee, S. Cho, and D. Kim, "Device mobility management in contentcentric networking," IEEE Communications Magazine, vol. 50, 2012.

[14] Y. Rao, H. Zhou, D. Gao, H. Luo, and Y. Liu, "Proactive caching for enhancing user-side mobility support in named data networking," in 7th International conference on Innovative Mobile and Internet Services in Ubiquitous Computing (IMIS'13), 2013.

[15] F. Bruno, M. Cesana, M. Gerla, G. Mauri, and G. Verticale, "Optimal content placement in icn vehicular networks," in 5th International Conference On Network of the Future (Nof'14), 2014.

[16] PARC, "Ccnx 1.0 protocol specifications roadmap," Nov 2013.

[17] G. Mauri and G. Verticale, "On the tradeoff between performance and user privacy in information centric networking," in New Technologies, Mobility and Security (NTMS), 2014 6th International Conference on, March 2014, pp. 1-5.

[18] BonnMotion, "A mobility scenario generation and analysis tool," http://sys.cs.uos.de/bonnmotion/.

[19] Z. Li, G. Simon, and A. Gravey, "Caching policies for in-network caching," in 21st International Conference on Computer Communications and Networks (ICCCN'12), 2012. 